\title{
АВТОМАТИЗАЦИЯ МЕЛКОМОТОРНЫХ ДЕЙСТВИЙ В ФУНКЦИОНАЛЬНОМ ОБУЧЕНИИ ПАЦИЕНТОВ С ОНМК В РАННЕМ ВОССТАНОВИТЕЛЬНОМ ПЕРИОДЕ
}

\author{
(О Шутеева Т.В. ${ }^{1}$, Никишина В.Б. ${ }^{2}$, Петраш Е.А. ${ }^{1}$, Черняховский О.Б. ${ }^{3}$ \\ ${ }^{1}$ Курский государственный медицинский университет (КГМУ) \\ Россия, 305041, Курская область, г. Курск, ул. К. Маркса, д. 3 \\ ${ }^{2}$ Российский национальный исследовательский медицинский университет \\ имени Н.И. Пирогова (РНИМУ им. Н.И. Пирогова) \\ Россия, 117997, г. Москва, ул. Островитянова, д. 1 \\ ${ }^{3}$ Национальный медицинский исследовательский центр реабилитации и курортологии \\ (НМИЦ РК) \\ Россия, 121099, г. Москва, ул. Новый Арбат, д. 32
}

\begin{abstract}
Целью исследования является изучение автоматизации мелкомоторных действий в функциональном обучении пациентов с острыми нарушениями мозгового кровообращения (ОНМК) в раннем восстановительном периоде.

Материалы и методы: общий объем выборки составил 41 пациента (в возрасте от 45 до 60 лет) с ОНМК по ишемическому типу, имеющих клинический диагноз: I63 - «Инфаркт мозга» (по МКБ-10). Оценка неврологического статуса осуществлялась с использованием шкалы NIHSS; степень когнитивных расстройств оценивалась по шкале MMSE. Организация исследования включала в себя три этапа: констатирующий (оценка исходной степени функциональных нарушений); формирующий (функциональное обучение); этап контрольного тестирования (оценка динамики показателей до и после прохождения функционального обучения). В исследовании были использованы следующие методы и методики: тест Френчай, набор функциональных нейропсихологических проб (проба «заборчик», фигуры Рея-Остеррица, проба «кулак-ребро-ладонь», проба на реципрокную координацию), аппаратно-программный комплекс «Визуальная медицина».
\end{abstract}

Результаты. В результате исследования выявлено улучшение функционального состояния ведущей руки, улучшение показателей динамического праксиса по критериям дифференциация и координация, улучшение показателей зрительно-конструктивных навыков, улучшение реципрокной координации по критериям темп, точность, дифференциация и координация. Полученные данные свидетельствуют о положительной динамике функционального состояния ведущей (правой) руки пациентов по темпово-динамическим, координационным и дифференцирующим основаниям. Последовательная активация произвольного и автоматизированного уровней организации движений через повторяемость произвольного запуска двигательных программ, основу которых составляют мелкомоторные действия, обеспечивает их автоматизацию.

Заключение. Возможность применяемого в функциональном обучении пациентов с ОНМК программноаппаратного комплекса «Визуальная медицина» визуальной демонстрации образцов выполнения как отдельных мелкомоторных действий, так и мелкомоторных действий, составляющих двигательную программу, обеспечивает переход от произвольного уровня организации движений к автоматизированному через механизм отзеркаливания.

Ключевые слова: автоматизация мелкомоторных действий, функциональное обучение, острые нарушения мозгового кровообращения, уровни организации движений.

Шутеева Татьяна Владимировна - канд. мед. наук, доцент, доцент кафедры неврологии и нейрохирургии, КГМУ, г. Курск. ORCID iD: 0000-0002-4009-2638. E-mail: ShuteevaTV@kursksmu.net

Никишина Вера Борисовна - д-р психол. наук, профессор, профессор кафедры организации непрерывного образования ФДПО, РНИМУ им. Н.И. Пирогова, г. Москва. ORCID iD: 0000-0003-2421-3652. E-mail: vbnikishina@mail.ru

Петраш Екатерина Анатольевна - д-р психол. наук, доцент, доцент кафедры психологии здоровья и нейропсихологии, КГМУ, г. Курск. ORCID iD: 0000-0002-3177-088X. E-mail: petrash@mail.ru (автор, ответственный за переписку)

Черняховский Олег Борисович - д-р мед. наук, зам. директора, НМИЦ PK, г. Москва. ORCID iD: 0000-0002-1769-4403. E-mail: Olegcrb2@mail.ru

По оценкам, в 2012 году от сердечнососудистых заболеваний в мире умерло 17,5 миллиона человек, что составило $31 \%$ всех случаев смерти в мире. Ежегодная смертность от инсульта в России составляет 374 на 100 тыс. населения и остается одной из наиболее высоких в мире. Одна треть выживших после инсульта достигает незначительных функциональных результатов через 5 лет после начала инсульта $[3,5,10]$. В клинической картине острых нарушений мозгового кровообращения (ОНМК) одним из ведущих проявлений являются нарушения двигательных функций (преимущественно мелкомоторных), которые, в свою очередь, обеспечивают успешность социальной адаптации пациентов и их социальное функционирование. 
Двигательные функции характеризуются иерархической структурой (включая рефлекторный, локомоторный, произвольный и автоматизированный уровни организации движений) и делятся на: функции поддержания позы тела (тоническая активность, реализуемая преимущественно медленными моторными единицами); собственно двигательные функции тела и его отдельных частей (фазная активность, обеспечиваемая преимущественно быстрыми моторными единицами). На рефлекторном уровне морфофункциональная организация движений обеспечивается активностью спинного мозга и стволовых отделов головного мозга; на локомоторном уровне включает в себя следующие структуры: субталамус, спинной мозг, красное ядро среднего мозга, ретикулярные ядра моста, вестибулярные ядра продолговатого мозга и моста, мозжечок и базальные ганглии. Морфофункциональная организация движений на произвольном уровне обеспечивается прецентральной извилиной лобной коры головного мозга, моторной корой, корой поясной извилины и ассоциативной лобной корой. Автоматизированный уровень движений обеспечивается базальными ганглиями, мозжечком и ассоциативной лобной корой.

Управление двигательными функциями также организовано иерархично. Морфологически система управления движениями обеспечивается на уровне структур спинного мозга, ствола головного мозга и корковых отделов головного мозга. Чем выше уровень сложности движений, тем более сложные программы управления реализуются. При выполнении какого-либо движения высшие центры запускают готовые моторные программы, заложенные (запрограммированные) в низших центрах, и формируют в конечном итоге из этих простых программ более сложные, которые в последующем автоматизируются.

В последние годы отмечается значительный прогресс в эффективности реабилитационновосстановительных мероприятий пациентов с ОНМК, включая применение методов медикаментозной терапии, а также различных методов физиовосстановления, включая, в том числе, применение автоматизированных и роботизированных методов. При этом акцент делается на восстановлении отдельных двигательных функций без учета их последующей автоматизации. Именно автоматизация мелкомоторных действий обеспечивает восстановление двигательных программ, запуск и реализация которых позволяют восстанавливать навыки самообслуживания.

Так, терапия вынужденными движениями, как один из методов восстановления двигатель- ных функций, подтвердила свою эффективность в работе с пациентами с ОНМК со стойкими нарушениями движений через 3-9 месяцев от начала заболевания [13]. Процедура данного вида терапии представляет собой активную тренировку паретичной конечности при искусственной иммобилизации неповрежденной руки, что позволяет отойти от привычного неиспользования паретичной руки [8]. Е.В. Костенко, Л.В. Петрова, А.В. Лебедева, А.Н. Бойко (2013), рассматривая комплексную реабилитацию пациентов с постинсультной спастичностью руки в амбулаторно-поликлинических условиях, установили, что применение ботулотоксина типа А (Ксеомин) в комплексной реабилитации больных с постинсультной спастичностью облегчает проведение кинезитерапии, ускоряет восстановление навыков самообслуживания, повышает качество жизни пациентов [4]. В.И. Тетиевский, А.А. Критари (2014) указывают на эффективность в комплексе с другими реабилитационными мероприятиями специально разработанных индивидуализированных программ гидротерапии, которые позволяют успешно противостоять развитию патологического процесса у пациентов, перенесших ОНМК. Данный метод, по мнению авторов, способствует также выраженному улучшению психоэмоционального состояния пациента за счет релаксации и седативного эффекта [9]. Исследователи также указывают на эффективность физической реабилитации пациентов, перенесших ОНМК [1], в том числе и с применением автоматизированных и компьютеризированных методов и технологий (например, роботизированной механотерапии) [7].

Таким образом, основные принципы реабилитации и восстановления пациентов с ОНМК ориентированы преимущественно на частые и интенсивные тренировки, направленные на конкретные задачи, функциональный подход [11].

При этом анализ множества накопленных эмпирических данных подтверждает необходимость задействования всех структурнофункциональных уровней организации движений в реабилитационно-восстановительном процессе [12].

Функциональное обучение пациентов с ОНМК предполагает последовательную активацию локомоторного, произвольного и автоматизированного уровней организации движений через повторяемость произвольного запуска двигательных программ, основу которых составляют мелкомоторные действия [6]. В основании функционального обучения лежит механизм отзеркаливания. Зеркальные нейроны возбуждаются как при выполнении определен- 
ного действия, так и при наблюдении за выполнением этого действия. Активация (запуск) зеркальных нейронов обеспечивает построение и корректировку модели наблюдаемых действий, что, в свою очередь, позволяет обеспечить функции освоения как мелкомоторных навыков, так и навыков, связанных с речью. Применение программно-аппаратного комплекса «Визуальная медицина» основано на возможности визуальной демонстрации образцов выполнения как отдельных мелкомоторных действий, так и мелкомоторных действий, составляющих двигательную программу, с возможностью предоставления визуальной обратной связи (пациенту одновременно предъявляется визуальный образец выполнения действия с аудиальным сопровождением и результат его выполнения пациентом) (рис. 1).
Целью исследования является изучение автоматизации мелкомоторных действий в функциональном обучении пациентов с ОНМК в раннем восстановительном периоде.

\section{МАТЕРИАЛЫ И МЕТОДЫ ИССЛЕДОВАНИЯ}

Общий объем исследовательской выборки составил 41 пациент с ОНМК по ишемическому типу в возрасте от 45 до 60 лет $(54,29 \pm 3,51)$. Критерием включения испытуемых в исследовательские группы было наличие диагноза I63 «Инфаркт мозга» (по МКБ-10) с полушарной локализацией очага ишемического инсульта, ранний восстановительный период инсульта. Критериями исключения являлись: признаки внутричерепного кровоизлияния любой локализации, ишемический инсульт в вертебробазилярном бассейне, тяжелая сопутствующая

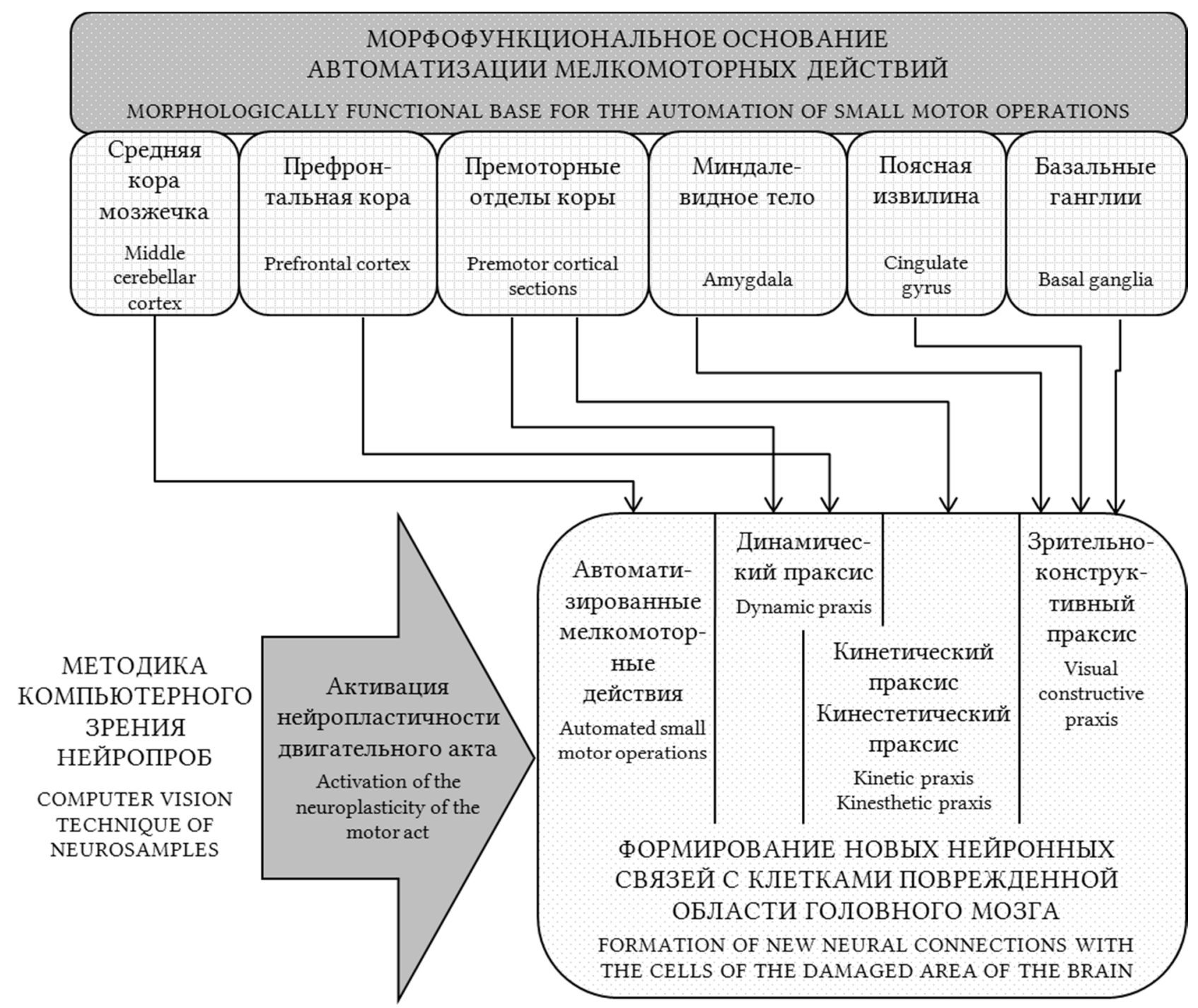

Рис. 1. Концептуально-методологическая схема исследования автоматизации мелкомоторных действий в функциональном обучении пациентов с ОНМК в раннем восстановительном периоде.

Fig. 1. Conceptual and methodological scheme for the study of automation of fine-motor actions in the functional training of patients with stroke in the early recovery period. 
соматическая патология, эпилепсия, психические расстройства. Характер очага поражения и инсульта определялся с использованием методов нейровизуализации - компьютерной томографии (КТ) и магнитно-резонансной томографии (МРТ). В неврологическом статусе пациентов с ОНМК выявлены неврологические нарушения средней либо легкой степени тяжести, их оценка проводилась по шкале NIHSS (National Institute of Health Stroke Scale); степень когнитивных расстройств оценивалась по MMSE (краткая шкала оценки психического статуса). У всех пациентов наблюдались моторные нарушения в виде гемипарезов на стороне контрлатеральной очагу поражения. Все пациенты уравнивались по уровню образования (имеют высшее образование).

Исследование проводилось на базах Курской областной клинической больницы (отделение для больных с острыми нарушениями мозгового кровообращения) и санатория «Соловьиные зори» (отделения долечивания постинсультных пациентов, куда пациенты поступали в раннем восстановительном периоде). Работа с пациентами велась в первой половине дня с 12.00 до 14.00 часов индивидуально в одном и том же помещении.

Организация исследования включала в себя три этапа: констатирующий, формирующий и этап контрольного тестирования. Задачей первого этапа являлась оценка исходного уровня функций кинетического, кинестетического, динамического, пространственного и конструктивного праксиса, а также профиля латеральной организации (в исследовании принимали участие пациенты, перенесшие ишемический инсульт, с левополушарным профилем латеральной организации - праворукие). Задачей второго этапа являлось функциональное обучение пациентов, перенесших ишемический инсульт, с использованием метода компьютерного зрения (программно-аппаратный комплекс «Визуальная медицина»). На третьем, завершающем, этапе осуществлялась сравнительная оценка показателей динамики восстановления автоматизации мелкомоторных действий в функциональном обучении пациентов с ОНМК в раннем восстановительном периоде до и после прохождения программы реабилитации (рис. 2).

В исследовании были использованы следующие методы и методики: тест Френчай, набор функциональных нейропсихологических проб (проба «заборчик», фигуры Рея-Остеррица, проба «кулак-ребро-ладонь», проба на реципрокную координацию), аппаратно-программный комплекс «Визуальная медицина». Аппаратнопрограммный комплекс представляет собой интерфейс пользователь-компьютер, технология машинного зрения которого осуществляется за счет получения изображения через веб-камеру. Камера фиксирует выполнения заданий пациентом, и компьютер в автоматическом режиме дает обратную связь.

Нейропсихологические пробы оценивались по шкале Л.И. Вассерман по критериям темпа, точности, дифференцированности и координированности. Каждой пробе были присвоены свои числовые значения в зависимости от качества выполнения пробы: 0 баллов (отсутствие ошибок или «неспецифические» ошибки для той или иной группы, которые являются свойственными для здоровых испытуемых); 1 балл (несформированность функции слабовыраженного характера в виде мелких погрешностей, которые исправляются самим испытуемым без участия экспериментатора); 2 балла (несформированность функции средней степени; испытуемый выполняет задание после нескольких попыток; выполняет задание без развернутых подсказок и наводящих вопросов); 3 балла (несформированность функции, которая проявляется даже после подробного многократного разъяснения инструкции экспериментатором, что приводит к невыполнению задания) [2].

Статистическая обработка была произведена с помощью программы Excel и STATISTICA 10. Сравнительная оценка показателей до и после прохождения функционального восстановления осуществлялась с использованием непараметрического критерия Вилкоксона $(\mathrm{p}<0,05)$. Сравнение показателей экспериментальной и контрольной групп после завершения этапа функционального восстановления осуществлялось с использованием непараметрического U-критерия Манна-Уитни $(\mathrm{p}<0,05)$.

\section{РЕЗУЛЬТАТЫ ИССЛЕДОВАНИЯ И ИХ ОБСУЖДЕНИЕ}

В результате исследования исходного набора двигательных реакций, включающих функции кинетического, кинестетического, динамического, пространственного и конструктивного праксиса, наиболее существенные нарушения были выявлены при реализации функций динамического, пространственного и конструктивного праксиса на уровне дифференциации и координации двигательного акта. Нарушение темповых характеристик проявлялось в долгой врабатываемости при выполнении проб (рис. 3).

Максимальное снижение у пациентов с ОНМК выявлено по показателям динамического и пространственного праксиса. Пациенты испытывали значительные затруднения при воспроизведении пространственного положения кистей рук как относительно внешних объектов 


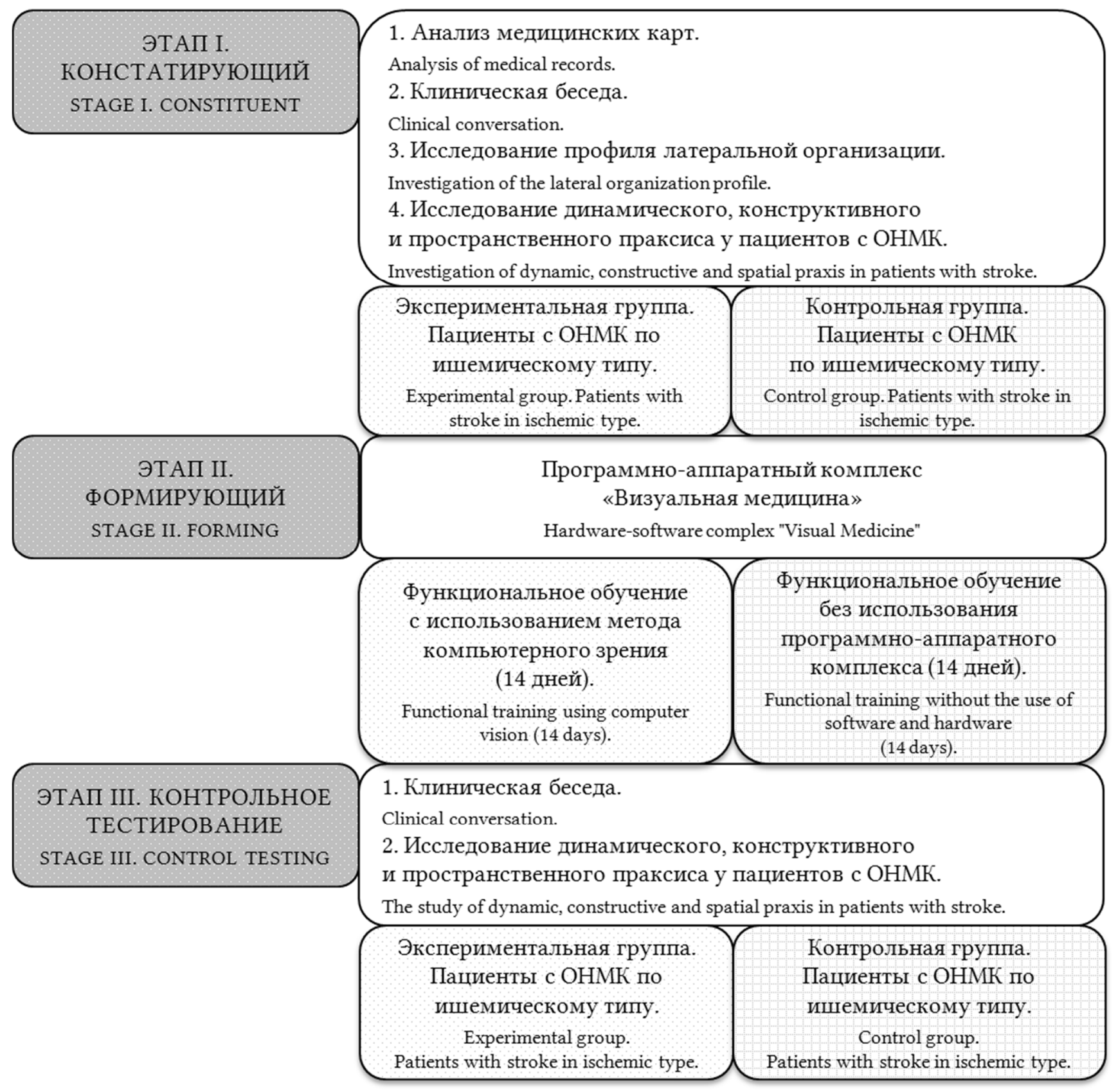

Рис. 2. Организация исследования автоматизации мелкомоторных действий в функциональном обучении пациентов с ОНМК в раннем восстановительном периоде.

Fig. 2. Organization of the study of automation of fine-motor actions in the functional training of patients with stroke in the early recovery period.

(нарушение пространственной ориентировки), так и относительно частей собственного тела (нарушение квазипространственной ориентировки). Нарушение реципрокной координации, характеризующей динамический праксис, проявляется в отсроченном оттормаживании предшествующего действия (одновременное выполнение двух действий-антагонистов).

В результате оценки значимости различий (непараметрический U-критерий Манна-Уитни, $\mathrm{p}<0,05)$ в экспериментальной и контрольной группах статистически значимых различий не выявлено.
При исследовании показателей реальных и символических предметно-манипулятивных действий, основу которых составляют автоматизированные мелкомоторные движения, установлено, что пациенты с ОНМК испытывают значительные сложности в совершении мелкомоторных (реальных и символических) действий с предметами, связанные с необходимостью ориентирования руки относительно внешних предметов (например, взять стакан с водой), ориентирования предмета относительно себя (причесаться), ориентирования предмета относительно другого предмета (надеть прищепку на стержень) (рис. 4). 


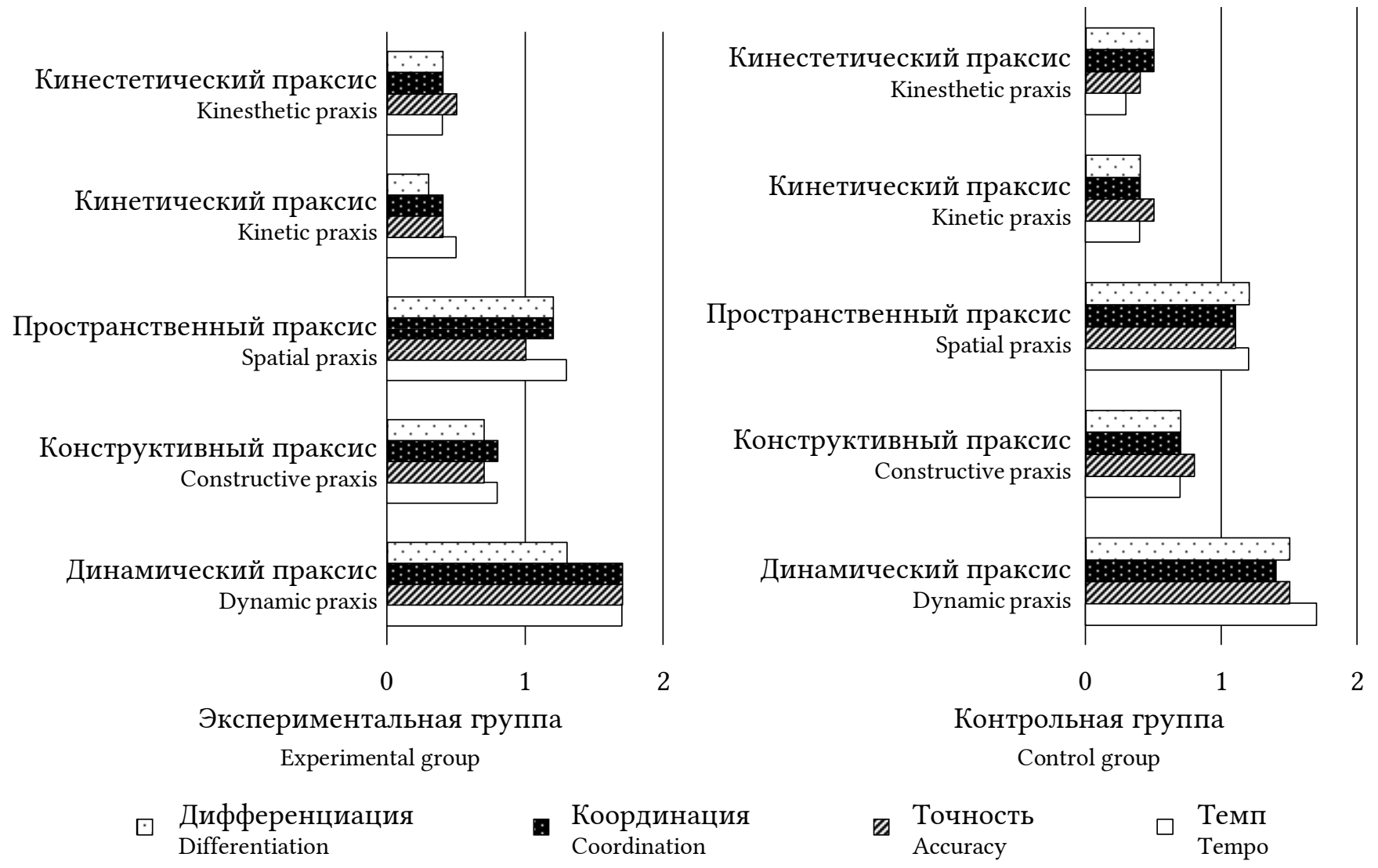

Рис. 3. Гистограммы средних значений показателей исходного уровня функций праксиса пациентов с ОНМК.

Fig. 3. Histograms of average values of indicators of the initial level of praxis functions of patients with stroke.

Тест Френчай
Frenchay arm test

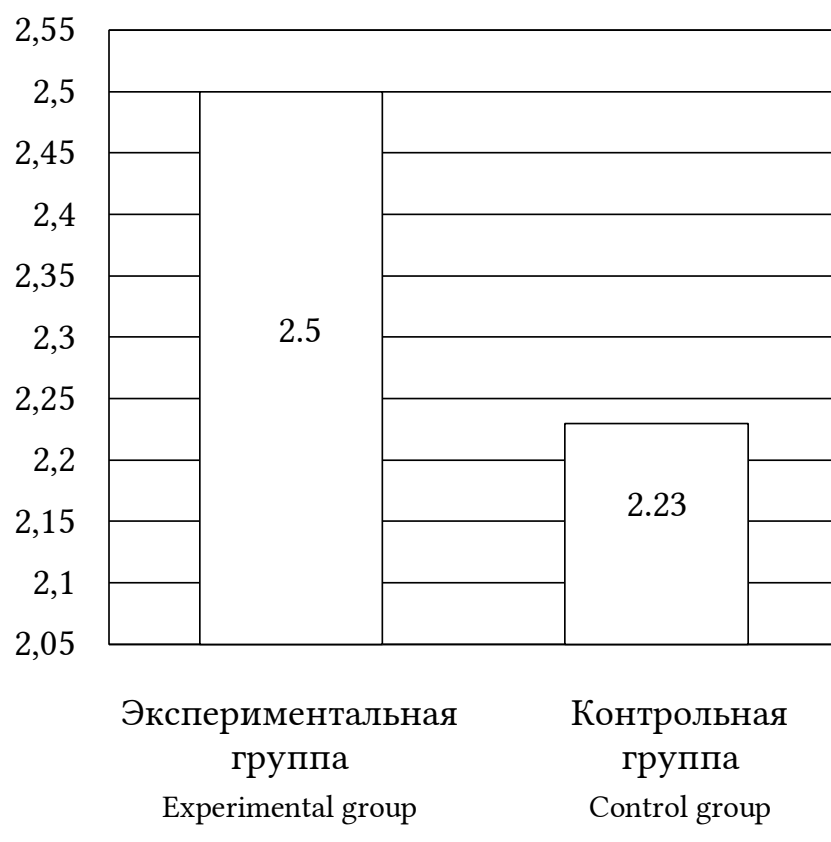

Показатели диагностики с использованием программно-аппаратного комплекса

Diagnostic indicators using hardware and software

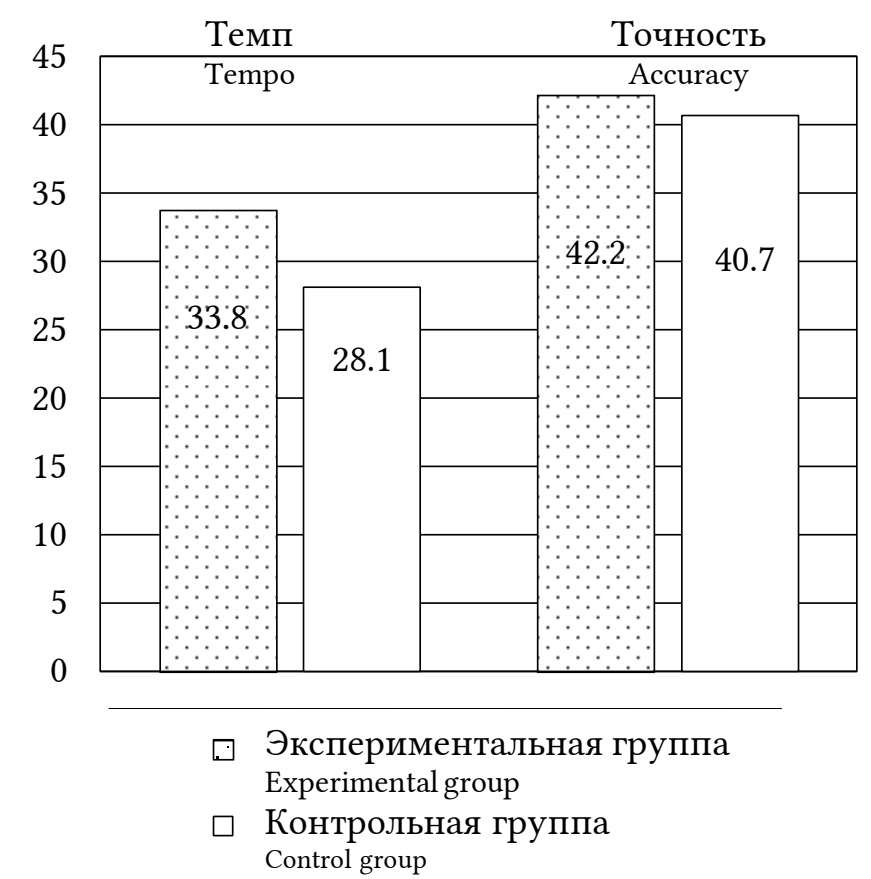

Рис. 4. Гистограммы средних значений показателей исходного уровня исследования манипулятивных возможностей рук.

Fig. 4. Histograms of average values of indicators of the initial level of researching the manipulative capabilities of hands. 
Курский научно-практический вестник "Человек и его здоровье". - 2019. - № 2.

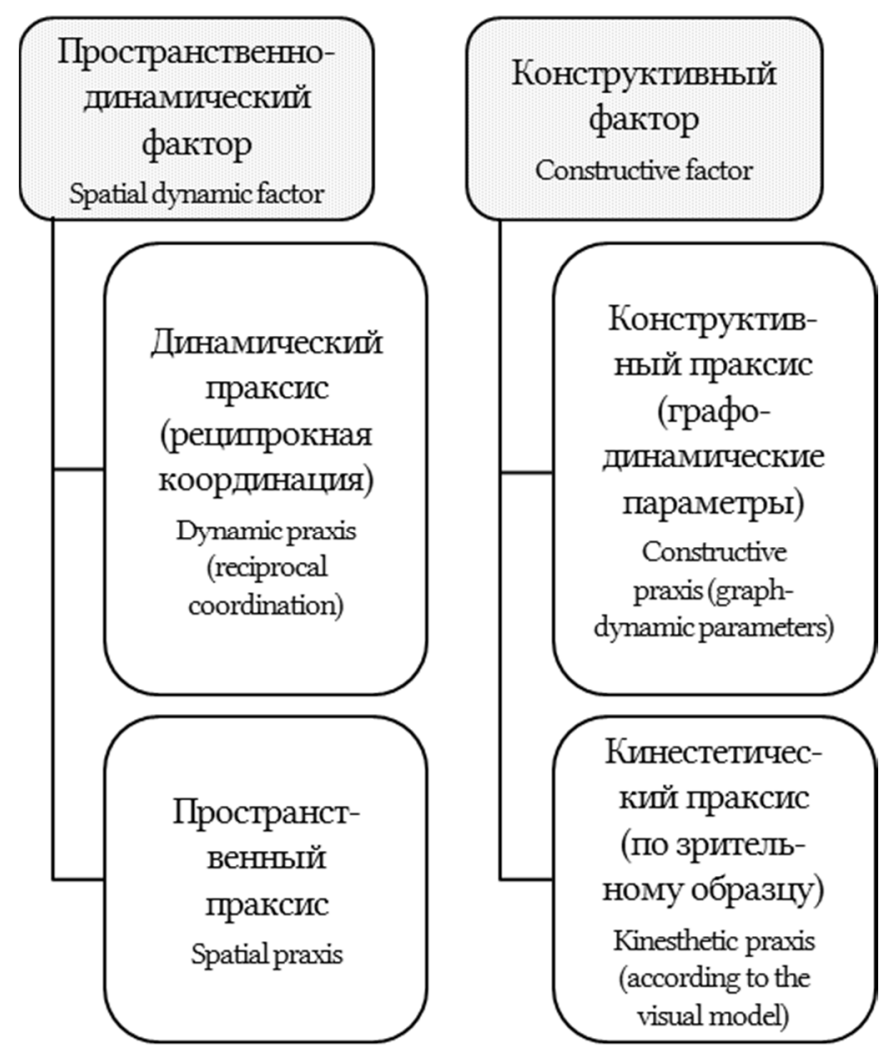

Экспериментальная группа

Experimental group

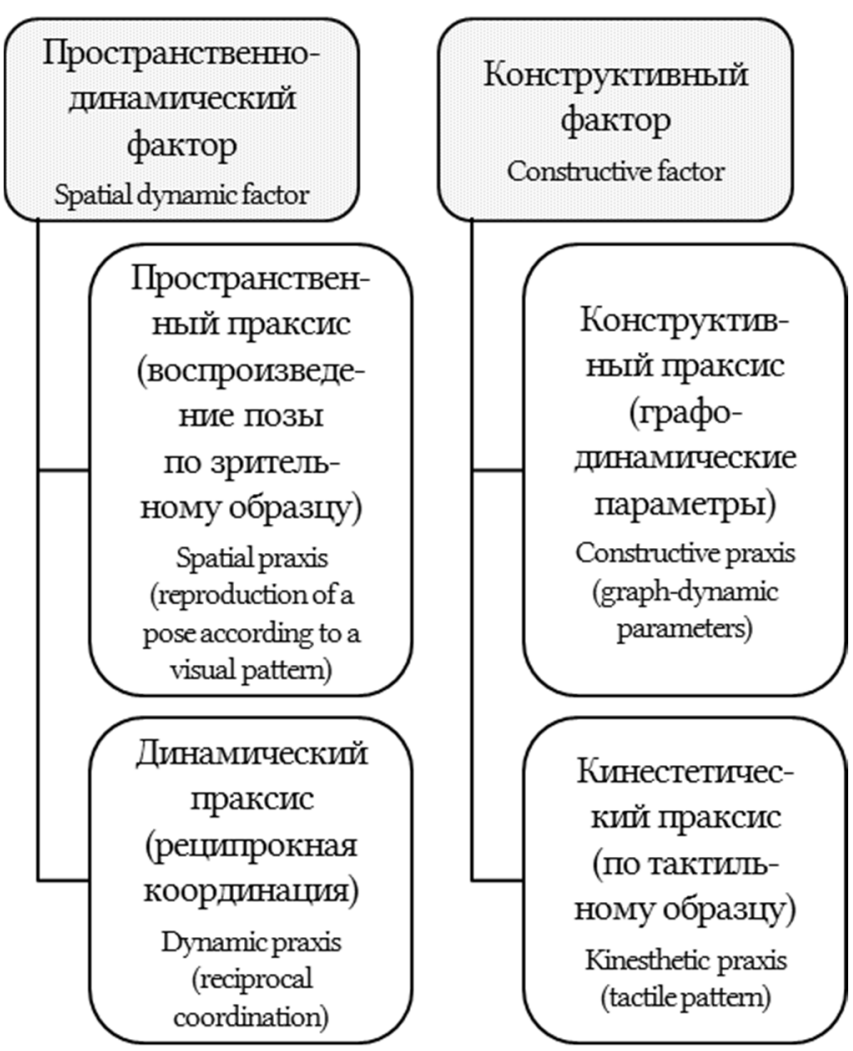

Контрольная группа

Control group

Рис. 5. Факторная структура мелкомоторных действий пациентов с ОНМК.

Fig. 5. Factor structure of fine-motor actions of patients with stroke.

При этом показатели диагностики исходного уровня пространственного, динамического и конструктивного праксиса с использованиемпрограммно-аппаратного комплекса «Визуальная медицина» соответствую данным, полученным при нейропсихологическом исследовании с использованием набора функциональных проб.

Оценивая факторную структуру мелкомоторных действий пациентов с ОНМК (через процедуру факторного анализа с varimaxвращением, р<0,05), выявлено два фактора: пространственно-динамический и конструктивный в обеих группах испытуемых. В экспериментальной группе пространственно-динамический фактор включает в себя показатели реципрокной организации $(-0,673)$ и пространственного праксиса (-0,654). Конструктивный праксис представлен снижением графодинамических показателей $(-0,729)$ и кинестетического праксиса по зрительному образцу $(-0,731)$ (рис. 5).

В контрольной группе пространственнодинамический фактор также включает в себя функции пространственного $(-0,721)$ и динамического $(-0,714)$ праксиса. Конструктивный фактор представлен показателями конструктивного $(-0,624)$ и кинестетического праксиса $(-0,612)$.
Таким образом, нарушения пространственно-динамического и конструктивного факторов приводят к снижению автоматизации мелкомоторных действий пациентов с ОНМК и, как следствие, нарушению выполнения и невозможности последующей автоматизации двигательных программ.

Выявленные нарушения факторной структуры мелкомоторных действий пациентов с ОНМК были заложены в основу программы функционального обучения с использованием программно-аппаратного комплекса «Визуальная медицина», которую проходили пациенты экспериментальной группы. Пациенты контрольной группы проходили восстановительное обучение с использованием традиционных методов, включающих функциональные нейропсихологические пробы.

В результате повторной оценки функций праксиса у пациентов с ОНМК с использованием программно-аппаратного комплекса «Визуальная медицина» выявлена положительная динамика восстановления мелкомоторных действий в процессе функционального обучения (рис. 6). 


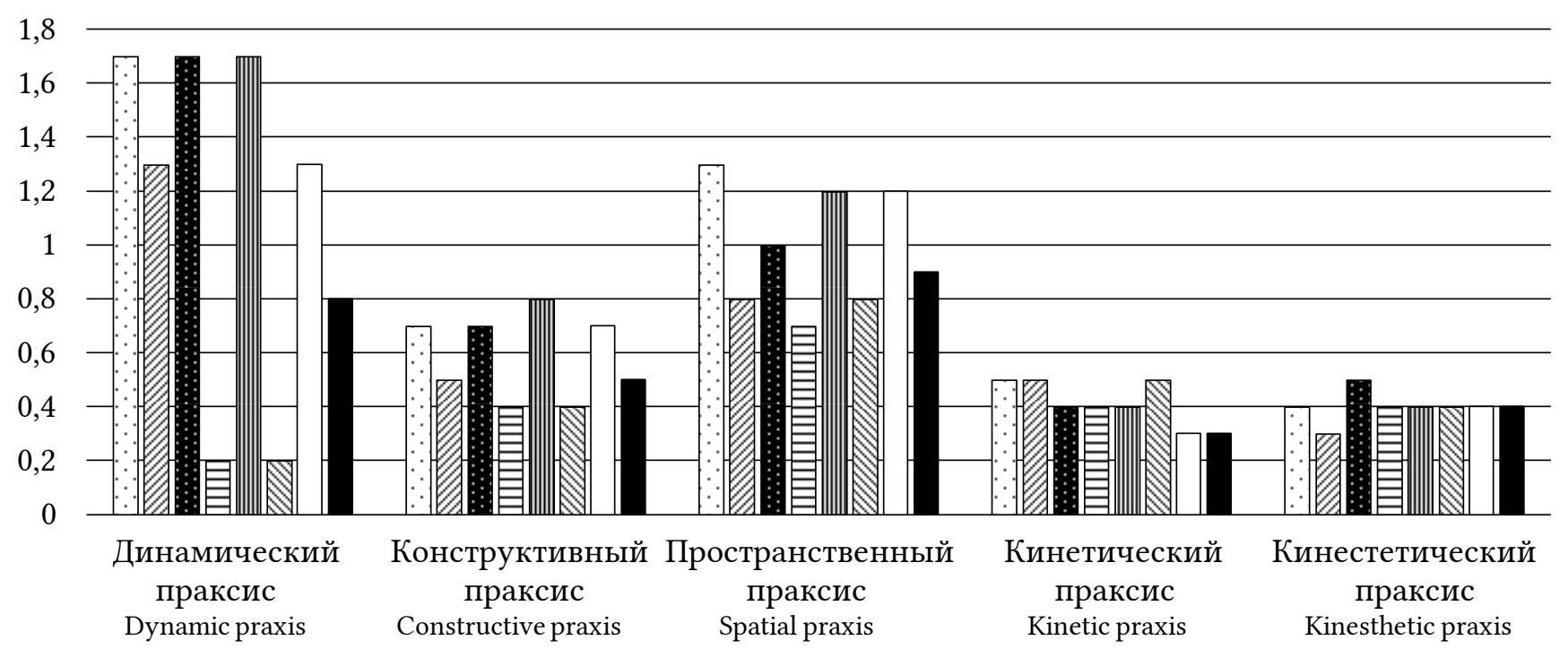

Экспериментальная группа

Experimental group

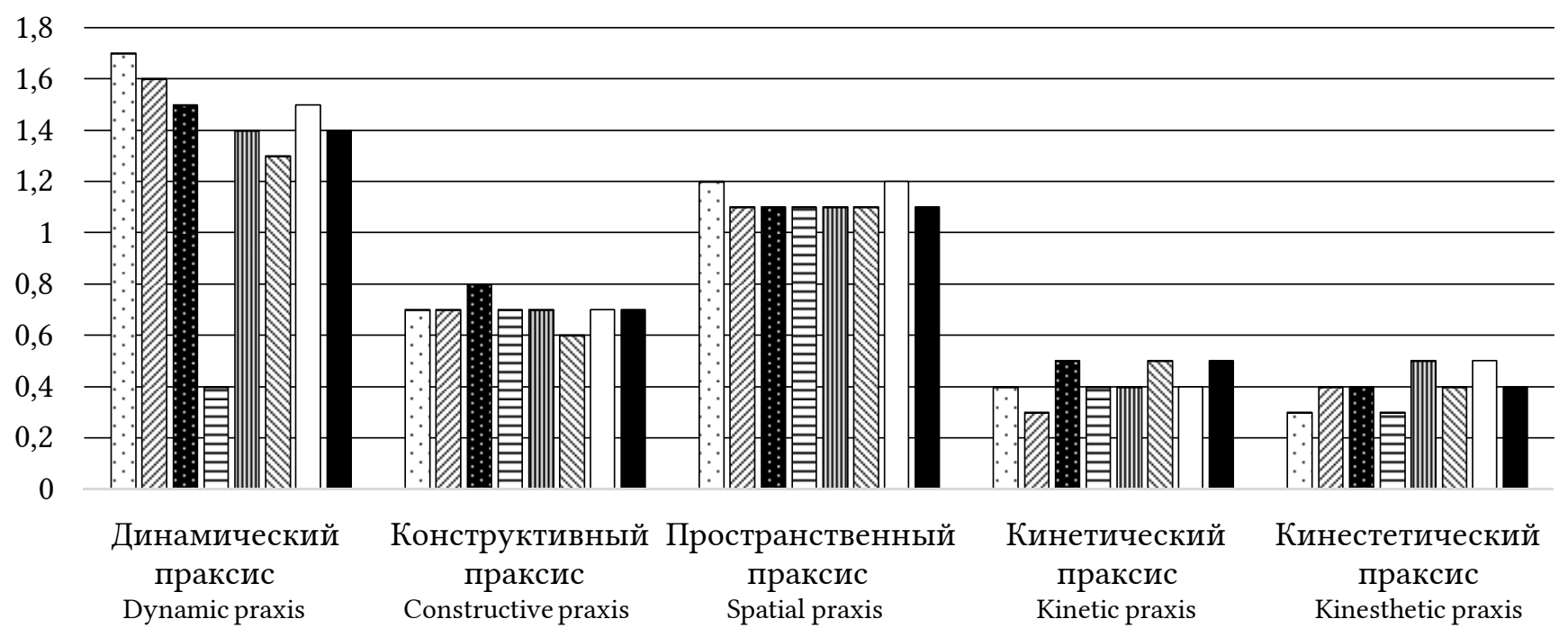

Контрольная группа

Control group
๑ Темп до
Tempo before
曰 Точность после Accuracy after
Дифференциация до Differentiation before

\section{च Темп после Tempo after}

m Координация до Coordination before

Дифференциация после Differentiation after

\section{Точность до Accuracy before \\ \& Координация после Coordination after}

Рис. 6. Показатели динамики восстановления функций праксиса у пациентов с ОНМК до и после функционального обучения.

Fig. 6. Indicators of the rescovery dynamics of praxis functions in patients with stroke before and af-ter functional training.

После завершения курса функционального обучения у пациентов с ОНМК в графодинамической пробе наблюдается нормализация линии рисунка в пространстве листа, уменьшение макро-/микрографий, уменьшений количества прерываний до 0-2, исчезновение персевераций элементов, что свидетельствует о тенденции к восстановлению функции динамического праксиса. При воспроизведении фигуры РеяОстеррица у пациентов с ОНМК после завершения программы функционального обучения наблюдается нормализация структурнотопологического отображения с сохранением целостности и пропорций как элементов, так и 
рисунка в целом, что свидетельствует о тенденции к восстановлению оптико-пространственных характеристик мелкомоторных действий. При выполнении функциональной пробы «кулак-ребро-ладонь» у пациентов наблюдается повышение темпово-динамических характеристик, уменьшение количества персевераций и ошибок воспроизведения пространственного положения элементов моторной программы. Полученные данные свидетельствует о тенденции к функциональному восстановлению динамического праксиса. Также отмечается повышение темповых характеристик и уменьшение компенсаторных действий, что свидетельствует о тенденции к восстановлению реципрокной координации мелкомоторных действий.

В показателях диагностических единиц программно-аппаратного комплекса «Визуальная медицина» отмечается улучшение функционального состояния мелкомоторных действий и их автоматизация на стороне поражения, увеличение темповых характеристик, а также повышение точности выполняемых проб эталонным предъявлениям (рис. 7).

Тест Френчай

Frenchay arm test

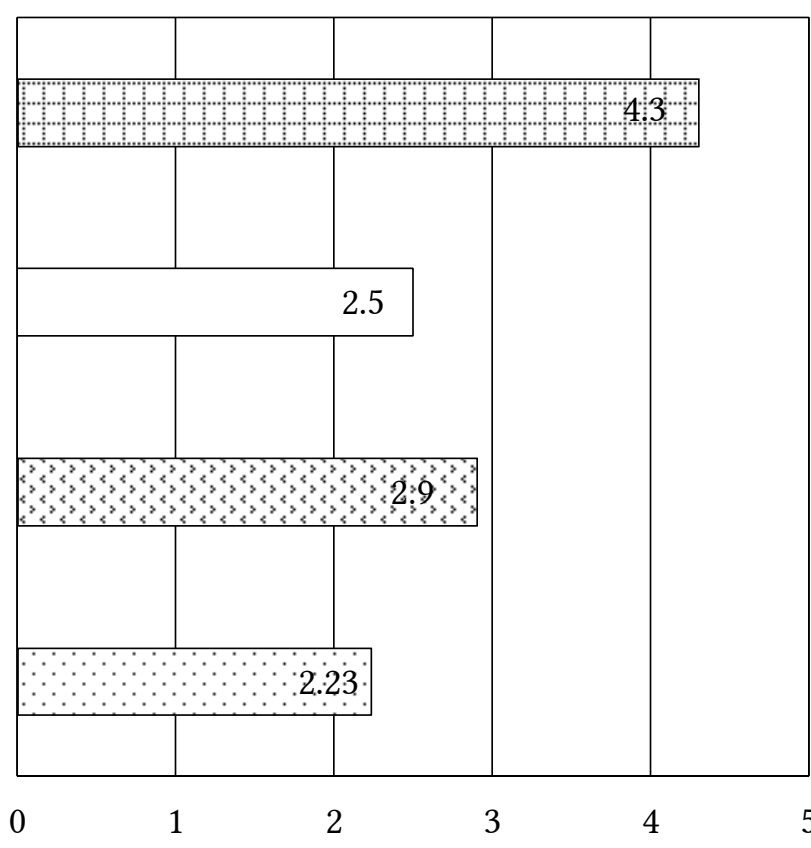

○ Экспериментальная группа после Experimental group after

๑ Контрольная группа после Control group after
Обобщая результаты исследования эффективности восстановления моторных функций у пациентов с ОНМК по ишемическому типу, выявлено: улучшение функционального состояния правой руки, улучшение показателей динамического праксиса по критериям дифференциация и координация, улучшение показателей зрительно-конструктивных навыков, улучшение реципрокной координации по критериям темп, точность, дифференциация и координация.

Таким образом, в результате исследования автоматизации мелкомоторных действий в функциональном обучении пациентов с ОНМК в раннем восстановительном периоде использование метода компьютерного зрения свидетельствует о положительной динамике функционального состояния ведущей (правой) руки пациентов по темпово-динамическим, координационным и дифференцирующим основаниям. Последовательная активация произвольного и автоматизированного уровней организации движений через повторяемость произвольного запуска двигательных программ, основу

Показатели диагностики с использованием программно-аппаратного комплекса

Diagnostic indicators using hardware and software

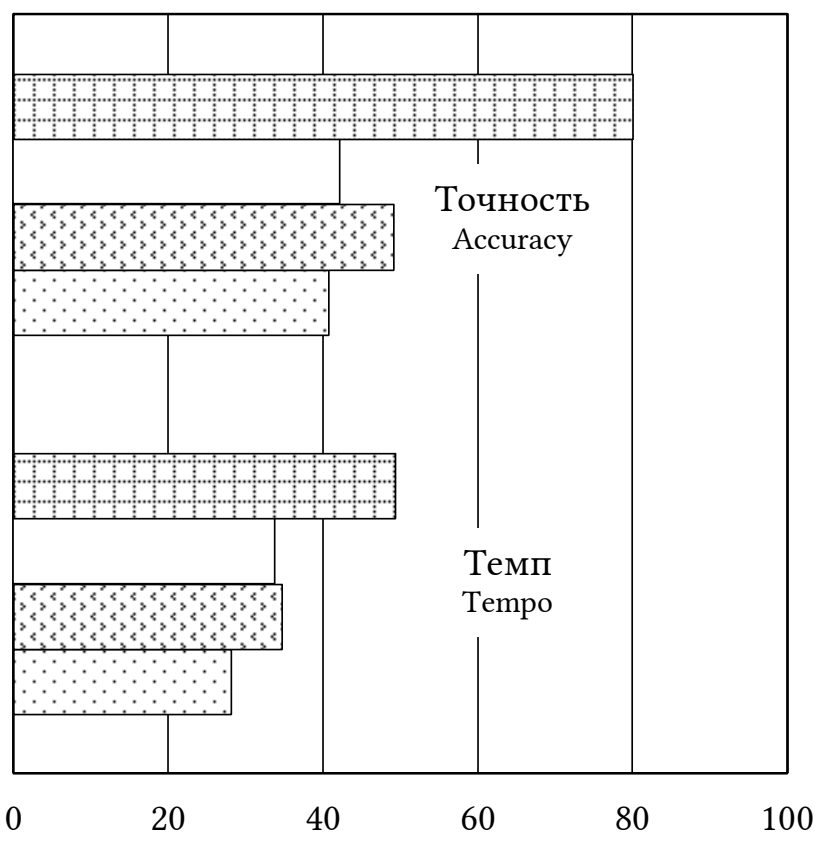

$\square$ Экспериментальная группа до Experimental group before

$\square$ Контрольная группа до Control group before

Рис. 7. Гистограммы средних значений показателей реальных и символических предметноманипулятивных действий до и после функционального обучения. ing.

Fig. 7. Histograms of average values of indicators of real and symbolic subject-manipulative actions before and after functional train- 
которых составляют мелкомоторные действия, обеспечивает их автоматизацию. Возможность применяемого в функциональном обучении пациентов с ОНМК программно-аппаратного комплекса «Визуальная медицина» визуальной демонстрации образцов выполнения как отдельных мелкомоторных действий, так и мелкомоторных действий, составляющих двигательную программу, обеспечивает переход от произвольного уровня организации движений к автоматизированному через механизм отзеркаливания.

\section{КОНФЛИКТ ИНТЕРЕСОВ}

Авторы декларируют отсутствие явных и потенциальных конфликтов интересов, связанных с публикацией настоящей статьи.

\section{ИСТОЧНИК ФИНАНСИРОВАНИЯ}

Авторы заявляют об отсутствии финансирования.

\section{СООТВЕТСТВИЕ ПРИНЦИПАМ ЭТИКИ}

Исследование проводилось в условиях информированного согласия, подписанного пациентами, и было одобрено 11.05.2018 г. Региональным этическим комитетом Курского государственного медицинского университета (протокол № 4).

\section{ЛИТЕРАТУРА/REFERENCES}

1. Архипов В.В., Привалов А.Н., Полойко А.А., Шестаков П.А. Опыт ранней физической реабилитации пациентов после ОНМК с использованием методики «S-E-T»-терапии на фоне применения нейропептидов. Российский медицинский журнал. 2010; 18(26): 1586-1590 [Arkhipov V.V., Privalov A.N., Poloyko A.A., Shestakov P.A. Experience of early physical rehabilitation of patients after CVA with the use of "SET"-therapy against the background of neuropeptides. Russian Medical fournal. 2010; 18(26): 1586-1590 (in Russ.)]

2. Вассерман Л.И., Дорофеева С.А., Меерсон Я.А. Mетоды нейропсихологической диагностики. Санкт-Петербург: Стройлеспечать, 1997. 360 с. [Vasserman L.I., Dorofeyeva S.A., Meyerson Ya.A. Methods of neuropsychological diagnosis. SaintPetersburg: Stroylespechat', 1997. 360 p. (in Russ.)]

3. Демографический ежегодник России. 2017: Статистический сборник. Москва: Росстат, 2017. 263 с. [Demographic Yearbook of Russia. 2017: a Statistical compendium. Moscow: Rosstat, 2017. 263 p. (in Russ.)]

4. Костенко Е.В., Петрова Л.В., Лебедева А.В., Бойко А.Н. Комплексная реабилитация пациентов с постинсультной спастичностью руки в амбулаторно-поликлинических условиях. Нервные болезни. 2013;3: 30-38 [Kostenko E.V., Petrova L.V., Lebedeva A.V., Boyko A.N. Comprehensive rehabilitation of patients with post-stroke spasticity of the arm in outpatient settings. Nervnyye bolezni. 2013; 3: 30-38 (in Russ.)]

5. Машин В.В. Инсульты: статистика и актуальность для нашей страны. Неврология и ревматология. Приложение к журналу Consilium medicum. 2014; 2: 24-25. [Mashin V.V. Strokes: statistics and relevance for our country. Nevrologiya i revmatologiya. Appendix to the journal Consilium medicum. 2014; 2: 24-25 (in Russ.)]

6. Никишина В.Б., Петраш Е.А., Шутеева Т.В., Никишин И.И., Никишина Е.И. Восстановительное обучение постинсультных пациентов методом нейропроб с помощью алгоритмов компьютерного зрения. Неврологический вестник. Журнал им. В.М. Бехтерева. 2018; 50(2): 83-88. [Nikishina V.B., Petrash E.A., Shuteeva T.V., Nikishin I.I., Nikishina E.I. Rehabilitation training method of post insult patients with the help of computer vision algorithms. Nevrologicheskiy vestnik. Zhurnal im. V.M. Bekhtereva. 2018; 50(2): 83-88 (in Russ.)]

7. Проказова П.Р., Пирадов М.А., Рябинкина Ю.В., Кунцевич Г.И., Гнедовская Е.В., Попова Л.А. Роботизированная механотерапия с использованием тренажера MOTOmed letto2 в комплексной ранней реабилитации больных с инсультом в отделении реанимации и интенсивной терапии. $А н_{-}$ наль клинической и экспериментальной невролоzuи. 2013; 7(2): 11-15 [Prokazova P.R., Piradov M.A., Ryabinkina Yu.V., Kuntsevich G.I., Gnedovskaya E.V., Popova L.A. Robotic mechanotherapy using the simulator MOTOmed letto2 in the complex early rehabilitation of stroke patients in the intensive care unit. Annaly klinicheskoy $i$ eksperimental'noy nevrologii. 2013; 7(2): 11-15 (in Russ.)]

8. Самосюк И.З., Фломин Ю.В., Самосюк Н.И., Пионтковская Н.И. Восстановление двигательных функций после инсульта: нейрофизиологические основы и мишени для реабилитационных вмешательств. Международный неврологический журнал. 2012; 8(54): 9-19. [Samosyuk I.Z., Flomin Yu.V., Samosyuk N.I., Piontkovskaya N.I. Motor Recovery after Stroke: Neurophysiological Basis and Targets for Rehabilitation Interventions. Mezhdunarodnyy nevrologicheskiy zhurnal. 2012; 8(54): 9-19 (in Russ.)]

9. Тетиевский В.И., Критари А.А. Индивидуализированные программы гидротерапии в условиях реабилитационного отделения для больных с острым нарушением мозгового кровообращения. Физиотерапия, бальнеология и реабилитация. 2014; 1: 55-56 [Tetievsky V.I., Kritari A.A. The individualized programs of hydrotherapy under conditions of a rehabilitation department for the patients presenting with acute disturbances of cerebral circulation. Fizioterapiya, Bal'neologiya i Reabilitatsiya. 2014; 1: 55-56 (in Russ.)]

10. Ярош А.С., Пирогова Л.А., Филина Н.А. Современное состояние проблемы острых нарушений мозгового кровообращения. Журнал Гродненского государственного медицинского университета. 2014; 3(47): 17-20. [Yarosh A.S., Pirogova L.A., Philina N.A. Current status of the problem of acute disorders of cerebral circulation. Fournal of the Grodno state medical university. 2014;3(47): 17-20 (in Russ.)] 
11. Dewey H.M., Sherry L.J. Stroke rehabilitation 2007: what should it be? Int $\mathcal{F}$ Stroke. 2007; 2(3): 191-200. DOI: $10.1111 / \mathrm{j} .1747-4949.2007 .00146 . x$.

12. Smith L.N., James R., Barber M., Ramsay S., Gillespie D., Chung C., Guideline Development Group. Rehabilitation of patients with stroke: summary of SIGN guidance. BMF. 2010; 340: c2845. DOI: 10.1136/bmj.c2845.

13. Nudo R.J., Barbey S. The mechanisms and neurophysiology of recovery from stroke. In: Harvey R.L., Stein J., Winstein C.J., Wittenberg G., Zorowitz R.D. Stroke re- covery and rehabilitation. Ney York: Demos Medical Publishing, LLC, 2009. p. 123-134. DOI: $10.1891 / 9781617051708.0007$

14. Wolf S.L., Winstein C.J., Miller J.P., Taub E., Uswatte G., Morris D., Giuliani C., Light K.E., NicholsLarsen D.; EXCITE Investigators. Effect of constraintinduced movement therapy on upper extremity function 3 to 9 months after stroke: the EXCITE randomized clinical trial. FAMA. 2006; 296(17): 2095-2104. DOI: 10.1001/jama.296.17.2095.

Для цитирования: Шутеева Т.В., Никишина В.Б., Петраш Е.А., Черняховский О.Б. Автоматизация мелкомоторных действий в функциональном обучении пациентов с ОНМК в раннем восстановительном периоде. Курский научно-практический вестник «Человек и его здоровье». 2019;(2):39-50. DOI: 10.21626/vestnik/2019-2/04.

\title{
AUTOMATION OF FINE MOTOR ACTIONS IN THE FUNCTIONAL TRAINING OF PATIENTS WITH STROKE IN THE EARLY RECOVERY PERIOD
}

\author{
○ Shuteeva T.V., Nikishina V.B. ${ }^{2}$, Petrash E.A. ${ }^{1}$, Chernyakhovsky O.B. ${ }^{3}$ \\ ${ }^{1}$ Kursk State Medical University (KSMU) \\ 3, K. Marx St., Kursk, Kursk region, 305041, Russian Federation \\ ${ }_{2}^{2}$ Pirogov Russian National Research Medical University (RNRMU) \\ 1, Ostrovityanova St., Moscow, 117997, Russian Federation \\ ${ }^{3}$ National Medical Research Center for Rehabilitation and Balneology (NMRC RB) \\ 32, Novy Arbat St., Moscow, 121099, Russian Federation
}

\begin{abstract}
Objective. The aim of the research is to study the automation of fine motor skills in the functional training of patients with stroke in the early recovery period.

Materials and methods: the total selection amount was 41 patients (aged 45 to 60 years) with ischemic type of stroke with a clinical diagnosis: I63 - "Brain infarction" (according to ICD-10). Neurological status was assessed using the NIHSS scale; the degree of cognitive impairment was assessed by the MMSE scale. The organization of the study included three stages: ascertaining (assessment of the initial degree of functional impairment); formative (functional learning); the stage of control testing (assessment of the dynamics of indicators before and after the passage of functional training). The study used the following methods and techniques: Frenchay arm test, a set of functional neuropsychological tests ("fence" test, ReyOsterrieth complex figure test (ROCF), "fist-palm edge-palm" test, reciprocal coordination test), "Visual Medicine» hardware-software complex.

Results. The study revealed an improvement in the functional state of the leading hand, an improvement in dynamic praxis indicators according to the differentiation and coordination criteria, an improvement in visual-constructive skills, an improvement in reciprocal coordination according to the criteria of tempo, accuracy, differentiation, and coordination. The obtained data testify to the positive dynamics of the functional state of the leading (right) hand of patients according to the tempo-dynamic, coordination and differentiating bases. Sequential activation of voluntary and automatic movements through the repeatable arbitrary start of motor programs, which are based on fine-motor actions, ensures their automation.

Conclusion. The possibility of using software-hardware complex "Visual Medicine", namely visual demonstration of patterns of both individual fine-motor actions and fine-motor actions that make up the motor program, used in functional training of patients with stroke, provides for the transition from a voluntary level of movements to an automatic one through the mirroring mechanism.

Keywords: automation of fine motor actions, functional training, acute disorders of cerebral circulation, levels of organization of movements.

Shuteeva Tatyana V. - PhD in Medicine, Associate Professor, Associate Professor of Neurology and Neurosurgery Department, KSMU, Kursk, Russian Federation. ORCID iD: 0000-0002-4009-2638. E-mail: ShuteevaTV@kursksmu.net

Nikishina Vera B. - Doctor of Psychology, Professor, Professor of the Department of Organization of Continuing Education FDPO, RNRMU, Moscow, Russian Federation. ORCID iD: 0000-0003-2421-3652. E-mail: vbnikishina@mail.ru

Petrash Ekaterina A. - Doctor of Psychology, Associate Professor, Associate Professor of Health Psychology and Neuropsychology Department, KSMU, Kursk, Russian Federation. ORCID iD: 0000-0002-3177-088X. E-mail: petrash@mail.ru (correspondence author)

Chernyakhovsky Oleg B. - DM, Deputy Director, NMRC RB, Moscow, Russian Federation. ORCID iD: 0000-0002-1769-4403. E-mail: Olegcrb2@mail.ru
\end{abstract}




\section{CONFLICT OF INTEREST}

The authors declare the absence of obvious and potential conflicts of interest related to the publication of this article.

\section{SOURCE OF FINANCING}

The authors state that there is no funding for the study.

\section{CONFORMITY WITH THE PRINCIPLES OF ETHICS}

The research was conducted under the con-ditions of informed consent signed by patients and was approved by the Regional Ethical Committee under Kursk State Medical Universi-ty of 11.05.2018 (Protocol No. 4).

Received 17.04.2019

Accepted 20.06.2019

For citation: Shuteeva T.V., Nikishina V.B., Petrash E.A., Chernyakhovsky O.B. Automation of fine motor actions in the functional training of patients with stroke in the early recovery period. Kursk Scientific and Practical Bulletin "Man and His Health". 2019;(2):39-50. DOI: $10.21626 /$ vestnik/2019-2/04. 